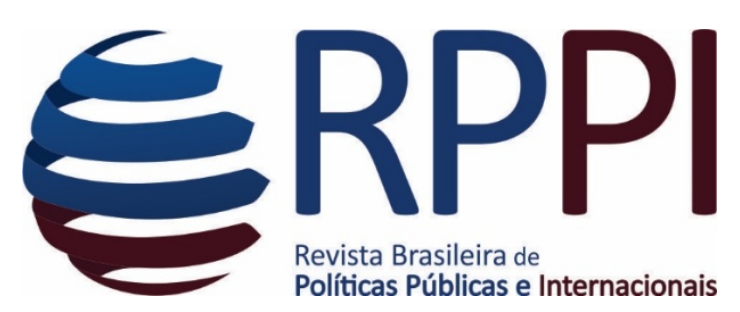

Data de recebimento: 23/07/2018

Data de aceite: 23/08/2018

Organização: Programa de Pós-Graduação em

Gestão Pública e Cooperação Internacional

(PGPCI/UFPB)

Avaliação: Por Pares.

\title{
As organizações internacionais na difusão de políticas de proteção à infância e adolescência no Brasil: um estudo de caso do Selo UNICEF - Município Aprovado
}

\section{International organizations on the diffusion of child and adolescent protection policies in Brazil: a case study of the UNICEF Seal - Approved Municipality}

\author{
Bruna Bezerra Oliveira \\ Universidade Federal de Pernambuco (UFPE) \\ Email: brunab.oliveira@icloud.com
}

\author{
Andrea Quirino Steiner \\ Universidade Federal de Pernambuco (UFPE) \\ Email: ecodea@gmail.com \\ Elia Elisa Cia Alves \\ Universidade Federal de Pernambuco (UFPE) \\ Email: eliacia@gmail.com
}

\begin{abstract}
Resumo: O seguinte trabalho trata da influência que as organizações internacionais podem exercer na política interna dos Estados, considerando a perspectiva da difusão de políticas públicas. O trabalho analisa a influência exercida pelo UNICEF na formulação de políticas públicas voltadas à infância e adolescência no Brasil, enfocando o programa Selo UNICEF - Município Aprovado. Assim, se caracteriza como estudo de caso único, em que se analisa o caso de uma organização internacional que difunde políticas públicas em meio a uma população de organizações (casos) com esse tipo de atuação no país. Entre os resultados principais, foi observado um processo de difusão por meio da atuação da organização através de seis mecanismos apresentados pela literatura (aprendizado, emulação, externalidades, coação, socialização e competição), bem como quatro dos cinco instrumentos na difusão de ideias, normas e políticas (disseminação de ideias, configurações padronizadas, funções coordenadoras e suporte técnico; o suporte financeiro não foi usado).
\end{abstract}

Palavras-chave: Organizações Internacionais, UNICEF, Políticas Públicas para a Infância, Interdependência, Difusão de Políticas.

Abstract: This study deals with the influence international organizations can have on countries' internal policy by considering the perspective of public policy diffusion. We analyze the influence of UNICEF on creating public policies aimed at children and teenagers in Brazil and focus on the program Selo UNICEF - Município Aprovado. Thus, it is a single case study, in which we analyze the case of one international organization that diffuses public policies among a population of organizations (cases) with 
OLIVEIRA, Bruna B.; STEINER, Andrea Q.; ALVES, Elia E. C. As organizações internacionais na difusão de políticas de proteção à infância e adolescência no Brasil: um estudo de caso do Selo UNICEF - Município Aprovado

this type of activity in the country. Among the main results we observed a policy diffusion process through the organization's activities, which used six of the diffusion mechanisms presented by the literature (learning, emulation, externalities, coercion, socialization and competition). In addition, we verified four out of the five diffusion instruments appointed by the literature on the diffusion of ideas, norms and policies (dissemination of ideas, standard settings, coordinating functions and technical support; financial support was not used).

Keywords: International Organizations, UNICEF, Public Policies for Children, Interdependence, Policy Diffusion.

\section{Introdução}

Este trabalho busca responder à seguinte pergunta: quais os mecanismos de difusão em atuação no processo de ampliação das políticas públicas brasileiras para a infância e adolescência promovidos pelo Fundo das Nações Unidas para a Infância - UNICEF? Como objetivo geral buscou-se verificar quais mecanismos de difusão de políticas internacionais se aplicam, com foco no caso do Selo UNICEF - Município Aprovado. Além disso, buscou-se: i) identificar os mecanismos de difusão de políticas internacionais revelados pela literatura, especificamente para as organizações internacionais (OIs) ${ }^{1}$ e ii) verificar quais mecanismos e instrumentos na difusão de ideias, normas e políticas se aplicam ao caso do Selo UNICEF Município Aprovado.

O texto está estruturado da seguinte maneira. Além desta seção introdutória, a segunda seção apresenta o referencial teórico e metodológico, no qual são discutidas algumas questões conceituais referentes à difusão de políticas e é realizada uma revisão dos principais mecanismos revelados pela literatura, com um foco na atuação das organizações internacionais, além do desenho de pesquisa e procedimentos utilizados. Na seção 3, é feita uma breve

\footnotetext{
${ }^{1}$ A literatura é diversa ao conceituar as organizações internacionais. Aqui optamos por uma definição mais restrita e pragmática, em que as organizações internacionais são vistas como "manifestações concretas das relações internacionais regularizadas", com "existência formal e material separada- apesar de dependente, em boa parte de Estados e grupos de dentro dos Estados” (ARCHER, 2001: p. 2). Desta forma, apesar do UNICEF ser formalmente uma agência da ONU, no texto o tratamos genericamente como organização, conforme o conceito supracitado.
}

Revista Brasileira de Políticas Públicas e Internacionais, v.3, n.2, Dezembro/2018, pp. 152-177. 
OLIVEIRA, Bruna B.; STEINER, Andrea Q.; ALVES, Elia E. C. As organizações internacionais na difusão de políticas de proteção à infância e adolescência no Brasil: um estudo de caso do Selo UNICEF - Município Aprovado

retrospectiva histórico-institucional do UNICEF e de sua relação com o Brasil, passando por acordos firmados, até a implementação do programa Selo UNICEF Município Aprovado. A seção 4 apresenta os resultados encontrados pela pesquisa, e o UNICEF e o Selo são analisados de acordo com a perspectiva da difusão. Aqui é possível observar se há ou não a presença de mecanismos de difusão no processo de implementação e nos mecanismos utilizados por esse programa. O trabalho encerra com algumas considerações finais e uma sugestão de agenda de pesquisa.

\section{Referencial teórico e metodológico}

\subsection{Difusão das políticas internacionais: questões conceituais}

Partindo do pressuposto da interdependência, não vamos aqui questionar se há influência entre as esferas nacional e internacional, mas sim, conforme sugere Putnam (1988), discutir alguns mecanismos através dos quais ocorre essa interação. Nos estudos das relações internacionais, as relações de influência mútua têm sido abordadas por diversos autores, entre os quais: Gourevitch (1978), Keohane \& Nye (1987), Putnam (1988), Milner (1997), Jakobi (2009), entre outros.

Nesta seção serão apresentados alguns dos conceitos propostos pela literatura quanto à difusão de políticas e seus mecanismos. O termo difusão "se refere a diferentes mecanismos causais que influenciam na adoção de políticas públicas” (Heize, 2011: p. 7); ou seja, esta linha de pesquisa estuda caminhos que são percorridos para que determinada política, ou as ideias acerca do tipo de política que deve ser implementada em determinado caso, sejam adotadas (Gilardi, 2016). Destaca-se que a difusão pode, ainda, ocorrer em diversas direções e entre diversos atores (Gilardi, 2012).

Em outras palavras, a difusão se preocupa com os processos que se desenvolvem em prol da implementação da política, e não com sua implementação de fato. É importante, também, atentar para os sentidos nos quais a difusão pode ocorrer. Pode ocorrer, por exemplo, no âmbito regional, onde se pode perceber a influência que as ações em uma unidade

Revista Brasileira de Políticas Públicas e Internacionais, v.3, n.2, Dezembro/2018, pp. 152-177. 
OLIVEIRA, Bruna B.; STEINER, Andrea Q.; ALVES, Elia E. C. As organizações internacionais na difusão de políticas de proteção à infância e adolescência no Brasil: um estudo de caso do Selo UNICEF - Município Aprovado

(município, Estado, etc.) provocam nas escolhas políticas de Estados vizinhos (Berry \& Berry, 1990; Volden, 2006). Estas, por sua vez, podem ser mediadas pelo comportamento de outros atores, como organizações internacionais, por exemplo (Simmons et al., 2006).

Há diferentes estudos a respeito de como se deve realizar uma análise dos processos de difusão, e acerca das diferentes formas pelas quais uma política pode ser difundida (Volden, 2006; Heinze, 2011; Maske \& Volden, 2011; Gilardi, 2012; Gilardi, 2016). Também há diversas interpretações e visões de como os diferentes mecanismos podem ser observados nos processos de difusão.

Uma definição mais ampla do que seria um mecanismo de difusão pode ser encontrada em Braun \& Gilardi (2006), que a define como constatações sistemáticas, sendo as mesmas capazes de explicar o comportamento de uma unidade em suas relações de influência com outra. Gilardi (2016) diz que há um consenso quanto à existência de três classes de mecanismos, sendo estas o aprendizado, a emulação e a competição. Já Heinze (2011) defende a existência de quatro classes: aprendizado, externalidades, emulação e socialização.

De acordo com Gilardi (2016), os mecanismos próprios do aprendizado são observados em situações nas quais o sucesso na implementação de dada política pública por um ator aumenta as chances de que um tipo semelhante seja adotado por outros. Já os mecanismos de emulação poderiam ser melhor observados em situações nas quais as políticas teriam mais chance de se difundir devido a um momento social considerado propício. Por fim, a competição se relacionaria à adequação e adoção de políticas por um agente que propõe alguma vantagem em favor próprio, em detrimento de outros agentes.

Em Heinze (2011), nos quatro tipos de mecanismos identificados, dois são relacionados com um comportamento mais ligado à lógica racionalista, enquanto os restantes são relacionados a um comportamento construtivista. No primeiro caso, estes são o aprendizado e a socialização, onde o aprendizado possui a mesma definição dada por Gilardi (2016) e a socialização é definida por situações nas quais o comportamento dos atores é guiado na medida em que eles passam a compartilhar crenças a partir da interação com outros atores. Os dois

Revista Brasileira de Políticas Públicas e Internacionais, v.3, n.2, Dezembro/2018, pp. 152-177. 
OLIVEIRA, Bruna B.; STEINER, Andrea Q.; ALVES, Elia E. C. As organizações internacionais na difusão de políticas de proteção à infância e adolescência no Brasil: um estudo de caso do Selo UNICEF - Município Aprovado

mecanismos subsequentes, externalidades e emulação, são os que ele relaciona à dimensão construtivista. As externalidades compõem um fenômeno que ocorre quando são postos incentivos no sentido de influenciar determinado tipo de política. Já a emulação ocorre quando os atores buscam aumentar a legitimidade de suas escolhas em termos de políticas públicas, se adequando a parâmetros difundidos na esfera internacional.

\subsection{Breve revisão da literatura de difusão de políticas}

O interesse na difusão de políticas não é novo. Segundo Gilardi (2012), esse tipo de ideia pode ser traçada desde meados do século XIX. Complementarmente, Graham et al. (2012) registram que aproximadamente 800 artigos científicos foram publicados sobre este assunto entre 1958 e 2008.

Graham et al. (2013) evidenciam, em revisão sistemática, a ampliação desta área de estudo e ressaltam, ainda, que muitos termos diferentes têm sido usados para se referir à difusão, tanto dentro da ciência política como em outras áreas. Dado esse longo histórico, podese observar uma ampla gama de estudo dedicados ao tema. Graham et al. (2013) dividem os estudos de difusão em quatro categorias: 1) política americana; 2) política comparada; 3) relações internacionais e 4) outros.

Dentre os diversos estudos sobre difusão, um dos trabalhos mais citados é o de Berry \& Berry (1990), que trata dos padrões de implementação de loterias estaduais nos Estados Unidos. Para realizar a pesquisa, os autores utilizaram o método de Análise Histórica de Eventos (AHE), um tipo de análise usada "explicar uma mudança qualitativa (um evento) que ocorre no comportamento de um indivíduo em determinado ponto no tempo” (Berry \& Berry, 1990: p. 398). Outro trabalho importante que faz uso da metodologia da AHE é o de Volden (2006), que foca a difusão de um programa de planos de saúde públicos para crianças de baixa renda nos Estados Unidos. Neste trabalho o autor faz uso da metodologia de AHE Ano-Díade (Dyad-Year EHA), na qual ele analisa pares de estados como se fossem uma só unidade.

Volden (2006), ao mencionar o método tradicional de AHE utilizado por Berry \&

Revista Brasileira de Políticas Públicas e Internacionais, v.3, n.2, Dezembro/2018, pp. 152-177. 
OLIVEIRA, Bruna B.; STEINER, Andrea Q.; ALVES, Elia E. C. As organizações internacionais na difusão de políticas de proteção à infância e adolescência no Brasil: um estudo de caso do Selo UNICEF - Município Aprovado

Berry (1990), diz que em estudos que utilizam esta metodologia "as adoções por parte dos estados podem ser influenciadas tanto por determinantes internos quanto por pressões externas de difusão, como, por exemplo, o fato de estados vizinhos já haverem ou não adotado a política”. Em seu estudo, apesar de reconhecer as possibilidades do método utilizado por Berry e Berry (1990), Volden (2006) faz uso das díades para determinar quais são os pares de estados que possuem maiores chances de copiar as políticas públicas uns dos outros.

No âmbito dos estudos internacionais de difusão, é possível destacar o trabalho de Weyland (2005), que pesquisou a difusão de reformas nos sistemas de pensão dos países da América Latina, iniciada a partir da adoção de um novo sistema de pensão no Chile. De acordo com o autor, a explicação que ele considera mais adequada para a difusão das reformas é através do método cognitivo-psicológico, que ajudaria a explicar o caráter de onda do fenômeno, assim como sua configuração territorial e a difusão de uma diversidade comum intermediária. Vale destacar um importante aspecto dos estudos de difusão, que é a questão territorial: em geral, se diz que a difusão ocorre entre unidades próximas.

Ainda na linha de estudos de difusão internacional, há os trabalhos de Jakobi (2005; 2009; 2012) nos quais a autora discorre sobre a difusão da ideia de aprendizagem contínua (long-life learning) na Europa. Através de uma análise de fatos históricos e relatórios, a autora demonstra a influência de organizações como a UNESCO nesse processo, além de elaborar um roteiro geral de como ocorreriam os processos de difusão na esfera internacional.

\subsection{O papel das organizações internacionais na difusão de políticas domésticas}

A literatura de difusão internacional admite influências mútuas entre diferentes unidades da política internacional. Gourevitch (1978), por exemplo, dedicou-se a estudar as pressões que a esfera internacional pode exercer na política interna dos países. Putnam (1988), por sua vez, atentou para a importância de compreender como e quando essas influências podem ocorrer. A partir da perspectiva da difusão, iremos discorrer brevemente sobre a literatura na qual se examina o papel que organizações internacionais podem desempenhar na

Revista Brasileira de Políticas Públicas e Internacionais, v.3, n.2, Dezembro/2018, pp. 152-177. 
OLIVEIRA, Bruna B.; STEINER, Andrea Q.; ALVES, Elia E. C. As organizações internacionais na difusão de políticas de proteção à infância e adolescência no Brasil: um estudo de caso do Selo UNICEF - Município Aprovado

política interna. Utilizaremos, no trabalho, a definição de difusão de Braun \& Gilardi (2006), em que a difusão é definida como "um processo no qual as escolhas são interdependentes, ou seja, onde as escolhas de um governo influenciam as escolhas feitas por outros e, consequentemente, as escolhas de um governo são influenciadas pelas escolhas feitas por outros” (p. 2).

Em geral, o papel das relações internacionais nos processos de difusão está diretamente ligado ao fenômeno espontâneo de entidades políticas de se adaptar às regras instituídas no meio internacional. Entretanto, em alguns textos, um dos mecanismos que é atrelado à difusão internacional de políticas, ideias e normas é a coação. Segundo essa perspectiva, as organizações internacionais fariam uso de poder para estimular a adoção de determinados tipos de política. Um exemplo seriam ações como a imposição de condicionalidades à concessão de empréstimos, práticas implementadas via organizações como o Banco Mundial e o Fundo Monetário Internacional - FMI (Gilardi, 2012).

Em outros trabalhos, tal como o de Heinze (2011), o papel de organizações internacionais pode ser atrelado a, pelo menos, três dos mecanismos supracitados: 1) a socialização, mecanismo através do qual se abriria um caminho para uma uniformização de regras, que seriam definidas como apropriadas a partir das interações entre os atores; 2) as externalidades, que se assemelham à coação, mas através do qual se oferece incentivos para os atores de forma a compeli-los a adotar dado tipo de política, podendo esses incentivos ser positivos ou negativos; e 3) a emulação, que seria “o desejo ou necessidade de atores domésticos de se adequar às normas espalhadas na esfera internacional” (Heize, 2011, p.12).

Segundo Jakobi (2009), a literatura sobre o papel das OIs na difusão de políticas ainda é incipiente. Para a autora, em geral, as organizações internacionais possuem a capacidade de gerar outputs no sistema político interno de países ao interagir com os mesmos. Neste sentido, cada organização traça diferentes caminhos para atingir tal objetivo, sendo os caminhos definidos pelos privilégios e status que essa possa ter. Na próxima seção, apresentam-se os principais elementos do trabalho dessa autora, expoente para o objeto de pesquisa em questão,

Revista Brasileira de Políticas Públicas e Internacionais, v.3, n.2, Dezembro/2018, pp. 152-177. 
OLIVEIRA, Bruna B.; STEINER, Andrea Q.; ALVES, Elia E. C. As organizações internacionais na difusão de políticas de proteção à infância e adolescência no Brasil: um estudo de caso do Selo UNICEF - Município Aprovado

bem como a metodologia aqui utilizada.

\subsection{A difusão e as organizações internacionais: aspectos metodológicos}

Os estudos de difusão são realizados com foco nos mais diferentes temas; assim, pode se observar na literatura uma ampla gama de metodologias aplicadas para atender às demandas dos diferentes tipos de trabalhos. Berry \& Berry (1990) iniciam seu artigo fazendo a descrição de duas linhas de pesquisa semelhantes utilizadas até então com bastante frequência nos estudos de difusão. Estas linhas envolvem análise transversal (cross-sectional analysis), nas quais a diferença se encontra numa variável dependente temporal; ou seja, o ano em que dada política foi adotada em cada unidade, ou o fato de a política haver sido ou não adotada em determinada data. Em seguida, os mesmos apresentam AHE como o método mais eficaz para analisar quais são os fatores que levariam à adoção de uma política em dado período de tempo.

Muitas vezes, os estudos focam a difusão por região, em agrupamentos (clusters), e seguindo um padrão de adoção através do tempo em forma de uma curva em "S”; ou seja, com início lento e, após certo tempo, aumentando consideravelmente o número de unidades a adotar a prática difundida (Berry \& Berry, 1990; Simmons \& Elkins, 2004; Graham et al., 2012). Apesar da frequência deste tipo de estudo, Weyland (2005) diz que, devido ao fato de haver inovações em várias partes do mundo, não haveria justificativa para fazer uso restrito de uma perspectiva regional. A questão da curva S também é posta em cheque, por não se adequar a casos nos quais há uma ampla adoção de um modelo logo após sua criação.

Maggetti \& Gilardi (2016) também atentam para o problema de mensurar os mecanismos de difusão. Ao analisar mais de uma centena de estudos da área, os autores encontraram diversas inconsistências, principalmente no que diz respeito aos estudos sobre emulação. Os autores indicam os três maiores problemas nos estudos analisados: falta de clareza conceitual, ausência de ligação entre os conceitos trabalhados e a mensuração aplicada e pouca atenção ao design da pesquisa.

Maggetti \& Gilardi (2016) ainda identificam os seis principais indicadores de difusão

Revista Brasileira de Políticas Públicas e Internacionais, v.3, n.2, Dezembro/2018, pp. 152-177. 
OLIVEIRA, Bruna B.; STEINER, Andrea Q.; ALVES, Elia E. C. As organizações internacionais na difusão de políticas de proteção à infância e adolescência no Brasil: um estudo de caso do Selo UNICEF - Município Aprovado

estudados pela literatura:

1) Proximidade geográfica - apesar de ser um componente importante da difusão, não pode ser ligado diretamente a nenhum mecanismo e é melhor estudado em conjunto com outros indicadores;

2) Filiação conjunta - participação (de um ator) em mais de uma instituição, organização ou grupo. Refere-se a contato direto e interação (independentemente de sua natureza), mas não à competição;

3) Sucesso das políticas - refere-se à operacionalização do sucesso de uma determinada política e pode ser ligada diretamente ao mecanismo de aprendizado;

4) Equivalência estrutural - identificação de unidades com posicionamento equivalente dentro de uma determinada rede, podendo ou não estas unidades estar competindo umas com as outras;

5) Número de adotantes prévios - se refere a quantas outras unidades adotaram a política em questão, em números absolutos ou relativos quanto a adotantes em potencial. Há uma possível ligação com o mecanismo de emulação devido às potenciais pressões normativas;

6) Fluxo comercial - pode indicar competição ou ligações mais genéricas entre unidades.

A autora oferece uma esquematização de como ocorreria o processo de formulação de políticas públicas levando em conta a influência da esfera internacional. Segundo ela, o processo contaria com seis estágios: 1) alinhamento dos fluxos de problemas, políticas públicas e política; 2) consequente entrada do tema na agenda; 3) como resultado, o sucesso das ideias centrais, afetando as etapas seguintes; 4) aplicação dos instrumentos pelas organizações internacionais; 5) difusão das políticas, devido à abertura da janela de oportunidades inicial (Jakobi, 2009).

Jakobi (2009) mostra o caminho através do qual as ideias disseminadas por organizações internacionais podem culminar em políticas públicas. O seu trabalho baseia-se principalmente em análises documentais, com o objetivo de mostrar os mecanismos de difusão

Revista Brasileira de Políticas Públicas e Internacionais, v.3, n.2, Dezembro/2018, pp. 152-177. 
OLIVEIRA, Bruna B.; STEINER, Andrea Q.; ALVES, Elia E. C. As organizações internacionais na difusão de políticas de proteção à infância e adolescência no Brasil: um estudo de caso do Selo UNICEF - Município Aprovado

observados para a ideia de aprendizagem contínua (lifelong learning). Em seu trabalho, há especial atenção direcionada para os mecanismos utilizados pelas organizações internacionais na difusão de políticas públicas (o estágio D do esquema apresentado acima). Quanto ao estágio B, a autora discorre sobre a janela de oportunidade que serve de ponto de partida para todo o processo de difusão, baseada na literatura de agenda setting de Kingdon (1995). A janela de oportunidade seria criada ao haver um alinhamento entre os fluxos de problemas, políticas e participantes denominados “visíveis”.

O fluxo de problemas se estabeleceria a partir do momento em que dada situação passasse a ser percebida como um problema. A seguir, o fluxo de políticas (policy) se daria diante da formulação de soluções para os problemas, onde se diz que essas seriam elaboradas através de discussões até que fosse atingido um consenso acerca da ação apropriada a ser tomada. Por fim, o último fluxo a ser alinhado seria o de participantes visíveis (politics), que consistiria nos atores interessados em tomar parte na solução do problema; assim, um problema que tenha maior atenção dos atores visíveis teria maior chance de fazer parte da agenda (Kingdon, 1995; Jakobi, 2009).

Quanto aos mecanismos de difusão de políticas internacionais, Jakobi (2009) sugere cinco processos nos quais as organizações internacionais buscam influenciar o processo de tomada de decisão nos Estados, a saber: 1) a disseminação discursiva, 2) a padronização de cenário, 3) os meios financeiros, 4) as funções coordenativas e 5) a assistência técnica.

A disseminação discursiva seria o instrumento através do qual as organizações internacionais conseguiriam colocar ideias específicas nas agendas políticas internacionais e “inclui regras ou suposições bem implícitas acerca de como se deveria lidar com problemas políticos” (Jakobi, 2009, p. 5).

Já a padronização de cenário seria um instrumento através do qual as organizações internacionais estabelecem padrões comuns de comportamento para os Estados, e convenções e acordos fazem parte desta categoria: "Padrões (...) não são apenas regras informais ou 'leis brandas’, mas também pode se referir a leis duras, como no caso de regulações dentro da União

Revista Brasileira de Políticas Públicas e Internacionais, v.3, n.2, Dezembro/2018, pp. 152-177. 
OLIVEIRA, Bruna B.; STEINER, Andrea Q.; ALVES, Elia E. C. As organizações internacionais na difusão de políticas de proteção à infância e adolescência no Brasil: um estudo de caso do Selo UNICEF - Município Aprovado

Europeia” (Jakobi, 2009, p. 5).

O uso de meios financeiros trata-se, simplesmente, do fornecimento de incentivos financeiros para que determinado tipo de política seja adotada. Neste sentido, Jakobi (2009) apresenta como exemplo os financiamentos do Banco Mundial, assim como a implementação de projetos na União Europeia.

Ainda segundo Jakobi (2009), “As funções coordenativas são instrumentos de vigilância, assim como de monitoramento informal” (p. 5). O papel desse instrumento é verificar se o país tem agido em prol de sua população de acordo com os preceitos aceitos na esfera internacional.

Quanto à assistência técnica, esse mecanismo pode ser utilizado de diferentes maneiras, de capacitações, a auxílio direto. Corresponde a ações diretas das organizações internacionais com objetivo de “...auxiliar ou aperfeiçoar as capacidades de um estado em relação aos seus objetivos em direção a um objetivo internacionalmente delineado e é comparável com programas de auxílio social, no nível nacional” (Jakobi, 2009, p.6).

Na análise da difusão do conceito de aprendizagem contínua, Jakobi (2009) se valeu da análise de documentos e relatórios de organizações internacionais, como a UNESCO, de forma a analisar o cumprimento das etapas do seu Modelo de Desenvolvimento Global de Políticas.

\subsection{Design de pesquisa e procedimentos}

O trabalho se caracteriza como estudo de caso único, em que se analisa o caso de uma organização internacional que difunde políticas públicas em meio a uma população de organizações (casos) com esse tipo de atuação no país, conforme Yin (2009). Optou-se por um estudo descritivo/exploratório (Gerring, 2012) devido à baixa quantidade de estudos sobre o papel das organizações internacionais na difusão de políticas públicas no país. Para tal atingir os objetivos propostos, foi analisada documentação oficial relativa às ações da organização em território brasileiro, através da ótica da difusão de políticas (policy diffusion).

Revista Brasileira de Políticas Públicas e Internacionais, v.3, n.2, Dezembro/2018, pp. 152-177. 
OLIVEIRA, Bruna B.; STEINER, Andrea Q.; ALVES, Elia E. C. As organizações internacionais na difusão de políticas de proteção à infância e adolescência no Brasil: um estudo de caso do Selo UNICEF - Município Aprovado

A escolha do UNICEF como caso se justifica por esta ser uma das organizações internacionais que atuam há mais tempo no Brasil, estando presente desde 1950 e influenciando a criação de leis importantes na sua área de atuação. Alguns exemplos são o artigo 227 da Constituição Federal e o Estatuto da Criança e do Adolescente. Entre suas ações, o Selo UNICEF - Município Aprovado é um caso interessante de ser analisado, por possuir diretrizes específicas a serem verificadas e pelo tempo de existência no país (desde 2004) (UNICEF, 2018).

A análise se baseia no arcabouço teórico proposto por Jakobi (2009) para estudar o papel das organizações internacionais na difusão de política, conforme apresentado anteriormente. A autora, por sua vez, considera o fato de que determinados instrumentos são utilizados pelas organizações internacionais com certa frequência, o que permite serem agrupados como instrumentos gerais de difusão.

Desta forma é feito, inicialmente, um relato histórico do UNICEF e de suas relações com o Brasil, até o desenvolvimento do programa Selo UNICEF - Município Aprovado. Posteriormente, o modelo desenvolvido pela autora é aplicado ao caso. Tanto a história da organização quanto suas ações são avaliadas, a fim de verificar a existência ou não de um processo de difusão em relação ao Selo para, em seguida, identificar os mecanismos.

\section{A difusão de políticas internacionais de proteção à infância e adolescência no Brasil: o caso do “Selo UNICEF - Município Aprovado”}

\subsection{Breve histórico da atuação do UNICEF no Brasil}

O Fundo das Nações Unidas para a Infância (UNICEF) foi fundado em uma sessão da Assembleia Geral das Nações Unidas, no ano de 1946, para promover o desenvolvimento e a segurança das crianças e adolescentes de todo o mundo. Criado no período pós Segunda Guerra Mundial, inicialmente o Fundo tinha como principal objetivo promover auxílio às crianças que sofriam com as adversidades causadas pelo conflito, provendo cuidados básicos e essenciais ao desenvolvimento. Com o passar dos anos seu campo de atuação foi ampliado, e atualmente a

Revista Brasileira de Políticas Públicas e Internacionais, v.3, n.2, Dezembro/2018, pp. 152-177. 
OLIVEIRA, Bruna B.; STEINER, Andrea Q.; ALVES, Elia E. C. As organizações internacionais na difusão de políticas de proteção à infância e adolescência no Brasil: um estudo de caso do Selo UNICEF - Município Aprovado

organização age em 191 países e territórios (UNICEF, 2018).

O UNICEF trabalha para garantir a proteção dos direitos da criança, ajuda-as a ter suas necessidades básicas atendidas e apresenta oportunidades para que elas descubram seus potenciais. Sua atuação é conduzida à luz da Declaração Universal de Direitos Humanos e da Convenção de Direitos da Criança. Os projetos da organização são realizados nos âmbitos mundial, regional e nacional. Atua junto aos governos para conseguir aprovação de leis e políticas públicas que estejam direcionadas para os problemas enfrentados pelas crianças e de proporcionar uma vida digna a elas. Trabalha nas arrecadações de fundos e recursos para ajudar países necessitados, principalmente aqueles em desenvolvimento (UNICEF, 2018).

Em setembro de 1990, na Cúpula Mundial para a Infância (World Summit for Children) que ocorreu em Nova Iorque, dois documentos foram adicionados à Convenção dos Direitos da Criança, de 1989. Nessa cúpula compareceram 152 líderes de Estado, representando quase a totalidade da população mundial. Lá, foi adotada a Declaração para a Sobrevivência, Proteção e Desenvolvimento da Criança, assim como um Plano de Ação para a implementação desta. Formou-se, assim, uma agenda para a infância, com objetivos para serem alcançados até o ano de 2000 (UNICEF, 1990).

Ao comprometerem-se com esta agenda, os líderes mundiais concordaram com o princípio do chamado First Call for Children, que coloca as crianças e adolescentes na faixa de prioridade no que diz respeito à formulação de políticas públicas e gasto de recursos. No documento, estabelecido em 30 de setembro do supracitado ano, a situação desafiadora das crianças no mundo é posta; fala-se dos anseios das crianças e de como, devido à situação global, estes se encontram em risco. O Brasil foi um dos signatários, comprometendo-se, então, a atender aos requisitos do documento (UNICEF, 2018).

Ao longo da história de seu relacionamento com o Brasil, a organização colaborou para que houvesse diversos avanços na qualidade de vida das crianças e jovens do país. O UNICEF fundou sua primeira base no território brasileiro em 1950, na cidade de João Pessoa, Paraíba, e desde então vem promovendo políticas de planejamento socioeconômico junto ao

Revista Brasileira de Políticas Públicas e Internacionais, v.3, n.2, Dezembro/2018, pp. 152-177. 
OLIVEIRA, Bruna B.; STEINER, Andrea Q.; ALVES, Elia E. C. As organizações internacionais na difusão de políticas de proteção à infância e adolescência no Brasil: um estudo de caso do Selo UNICEF - Município Aprovado

governo, de forma a priorizar a criança e o adolescente. Através da mobilização de grupos de interesse e do uso de pressão direcionada ao legislativo, para proteger juridicamente os direitos humanos e as garantias constitucionais para crianças e jovens, obteve sucesso em influenciar políticas públicas, como mencionado anteriormente.

Atualmente o foco da atuação no país é nas regiões Norte e Nordeste, uma vez que são nestas áreas onde se encontram as crianças em situação de maior risco. De forma a atendê-las melhor e para colaborar na realização dos Objetivos de Desenvolvimento do Milênio (ODM), em 2004 foi criado o Pacto Nacional Um Mundo para a Criança e Adolescente do Semiárido, que torna a ação nessa área prioritária, pacto este que foi ratificado em nível federal e posteriormente reafirmado no nível estadual pelos 11 estados da região Nordeste. Isso resultou na criação do programa Selo UNICEF - Município Aprovado. Na próxima seção, a implementação deste programa será discutida de maneira mais aprofundada.

\subsection{A implementação do Selo UNICEF - Município Aprovado}

Com a assinatura do Pacto Um Mundo para a Criança e o Adolescente do Semiárido em 2004, o programa Selo UNICEF - Município Aprovado foi ampliado para todos os estados do semiárido em 2005. Anteriormente havia sido aplicado no Ceará, o que serviu como um piloto para a ampliação do programa. O Selo é um programa que visa auxiliar os municípios na região do semiárido no Brasil, e foi desenvolvido pelo UNICEF com o objetivo maior de ajudar o país a alcançar os Objetivos de Desenvolvimento do Milênio relacionados às crianças e adolescentes. Assim, tal região foi escolhida por ser a que possui os piores indicadores sociais do país. Mais recentemente, o programa sofreu outra ampliação para abarcar, também, os municípios da região amazônica, outra detentora de baixos indicadores sociais.

Inicialmente o programa teve a duração de dois anos, sendo encerrado em 2006, ano no qual o UNICEF divulgou o primeiro relatório com os resultados alcançados por todos os 1.179 municípios participantes. O relatório conta com os resultados de todos os municípios e não apenas os aprovados, devido ao fato de todos haverem apresentado melhoras na maior

Revista Brasileira de Políticas Públicas e Internacionais, v.3, n.2, Dezembro/2018, pp. 152-177. 
OLIVEIRA, Bruna B.; STEINER, Andrea Q.; ALVES, Elia E. C. As organizações internacionais na difusão de políticas de proteção à infância e adolescência no Brasil: um estudo de caso do Selo UNICEF - Município Aprovado

parte dos indicadores. Um total de 192 municípios obteve o Selo nessa edição.

No relatório de resultados do Selo de 2008, apresentam-se os requisitos para a obtenção do Selo; assim, foram definidos 13 indicadores de impacto social e 21 indicadores de gestão. Era necessário que os municípios conquistassem avanços em ao menos, seis indicadores do primeiro grupo e dez do segundo, além de serem necessários avanços em dois temas de participação social, de um total de quatro. Na edição em questão, 1.130 municípios fizeram parte do programa, dos quais 259 conseguiram obter o Selo.

Na edição iniciada em 2009, o período de duração do programa foi ampliado para três anos como tempo durante o qual os municípios deveriam atingir suas metas para obter o Selo. Além disso, foi a partir desta edição que o programa passou a abarcar a região amazônica, passando a englobar 1.265 municípios do semiárido (dos quais 286 conseguiram o Selo) e 534 municípios da Amazônia (dos quais 121 conseguiram o Selo). A Tabela 1 traz uma perspectiva comparada dos números apresentados.

Tabela 1. Resultados do Programa Selo UNICEF - Município Aprovado.

\begin{tabular}{lccrrr}
\hline Duração & $\begin{array}{c}\text { Ano do } \\
\text { Selo }\end{array}$ & Região & $\begin{array}{c}\text { Municípios } \\
\text { inscritos }\end{array}$ & $\begin{array}{c}\text { Municípios que } \\
\text { obtiveram o selo }\end{array}$ & Percentual \\
\hline 2 anos & 2006 & Semiárido & 1.179 & 192 & $16,3 \%$ \\
2 anos & 2008 & Semiárido & 1.130 & 259 & $22,9 \%$ \\
3 anos & 2012 & Semiárido & 1.265 & 286 & $22,6 \%$ \\
& & Amazônia & 534 & 121 & $22,7 \%$ \\
3 anos & $2016^{*}$ & Semiárido & 1.134 & 308 & $27,2 \%$
\end{tabular}

*Os dados sobre a edição 2016 do selo não estavam disponíveis para a Amazônia no momento de elaboração desta tabela.

Na próxima seção os mecanismos do Selo UNICEF Município Aprovado serão analisados de acordo com a perspectiva da difusão, conforme explicado anteriormente.

Revista Brasileira de Políticas Públicas e Internacionais, v.3, n.2, Dezembro/2018, pp. 152-177. 
OLIVEIRA, Bruna B.; STEINER, Andrea Q.; ALVES, Elia E. C. As organizações internacionais na difusão de políticas de proteção à infância e adolescência no Brasil: um estudo de caso do Selo UNICEF - Município Aprovado

\subsection{O Selo e seus mecanismos de difusão}

Nesta seção tentaremos encontrar correspondências entre os mecanismos e instrumentos utilizados pelo UNICEF na difusão do programa Selo UNICEF - Município Aprovado e das políticas públicas direcionadas à infância e adolescência, e os mecanismos e instrumentos identificados pela literatura.

Assim, serão analisados documentos do UNICEF relacionados à sua interação com o Brasil, inicialmente em um contexto mais abrangente e, ao final, de forma mais focada no programa Selo UNICEF - Município Aprovado.

Ao colocar os mecanismos de difusão definidos por Heinze (2011) e Gilardi (2012;

2016) em contraste com os utilizados pelo UNICEF no programa e em suas relações com o Brasil, podemos observar vários pontos correspondentes (Quadro 01).

Quadro 01. Mecanismos utilizados na difusão de ideias, normas e políticas (conforme sugerido por Heinze, 2011; Gilardi, 2012 e Gilardi, 2016) e sua correspondência com o caso do Selo UNICEF - Município Aprovado.

\begin{tabular}{|c|c|}
\hline Mecanismo & $\begin{array}{c}\text { Observação para o Selo UNICEF - } \\
\text { Município Aprovado }\end{array}$ \\
\hline Aprendizado & $\begin{array}{l}\text { Divulgação de ações de sucesso dos } \\
\text { municípios nos Fóruns, incentivando outros } \\
\text { a adotar as medidas bem-sucedidas, ou } \\
\text { similares. }\end{array}$ \\
\hline Emulação & $\begin{array}{l}\text { World Summit for Children; First Call for } \\
\text { Children, Pacto um Mundo para a Criança e } \\
\text { o Adolescente do Semiárido. }\end{array}$ \\
\hline Externalidades e Coação* & $\begin{array}{l}\text { Exige a adoção de determinadas medidas } \\
\text { (condicionalidades) para que o município } \\
\text { participe do programa, como a existência de } \\
\text { um conselho municipal da criança e do } \\
\text { adolescente. }\end{array}$ \\
\hline Socialização & $\begin{array}{l}\text { Assemelha-se à emulação, no que diz } \\
\text { respeito aos meios, mas trata-se da adesão a } \\
\text { regras consideradas apropriadas, como as } \\
\text { difundidas pela UNICEF para a sociedade }\end{array}$ \\
\hline
\end{tabular}

Revista Brasileira de Políticas Públicas e Internacionais, v.3, n.2, Dezembro/2018, pp. 152-177. 
OLIVEIRA, Bruna B.; STEINER, Andrea Q.; ALVES, Elia E. C. As organizações internacionais na difusão de políticas de proteção à infância e adolescência no Brasil: um estudo de caso do Selo UNICEF - Município Aprovado

$\begin{array}{ll}\text { Competição } & \text { global } \\ \text { O próprio Selo como forma de premiação, } & \text { uma vez que a certificação ao final do } \\ \text { programa é um incentivo para a adoção das } \\ \text { medidas requeridas }\end{array}$

*Postos juntos devido à semelhança nos conceitos.

Fonte: elaboração das autoras.

Nos fóruns promovidos pelo Selo UNICEF - Município Aprovado, os municípios recebem informações sobre iniciativas bem-sucedidas que estão sendo realizadas nos municípios participantes. Essas informações facilitam a adoção de medidas semelhantes em seus próprios territórios. Nesse aspecto, podemos ver características do mecanismo de aprendizado.

A criação do Pacto um Mundo para a Criança e o Adolescente do Semiárido pode ser vista como uma forma de sensibilização das comunidades locais à necessidade do Brasil se adequar às regras e parâmetros reconhecidos internacionalmente. Essa busca por adequação aos padrões, reforçada pelos compromissos anteriores, assumidos no World Summit for Children e no ato da assinatura do First Call for Children, se encaixa na definição de mecanismos de emulação.

Para que o município esteja habilitado a participar do programa é necessário que o mesmo possua um Conselho Municipal da Criança e do Adolescente e, segundo o guia metodológico da última edição do programa, tornou-se obrigatória também a criação ou fortalecimento dos Núcleos de Cidadania dos Adolescentes (NUCA). A promoção de dois fóruns iniciais, sendo um de diagnóstico da situação do município e outro para a formulação de um plano de ação, também é necessária para que seja permitido ao município participar do programa. As exigências descritas enquadram-se na descrição de dois mecanismos de difusão, externalidades e coação.

Em contraste com o aprendizado e próximo da ideia de emulação, a socialização não está relacionada às consequências objetivas de uma política, mas com as características

Revista Brasileira de Políticas Públicas e Internacionais, v.3, n.2, Dezembro/2018, pp. 152-177. 
OLIVEIRA, Bruna B.; STEINER, Andrea Q.; ALVES, Elia E. C. As organizações internacionais na difusão de políticas de proteção à infância e adolescência no Brasil: um estudo de caso do Selo UNICEF - Município Aprovado

simbólicas, socialmente construídas a partir da atuação de um ator externo. Outra maneira de ver esse mecanismo é que o ônus da não adoção de determinadas regras internacionais pode mudar ao longo do tempo, como uma função de aceitação social. Assim, esse mecanismo pode ser identificado no esforço, por parte dos municípios, à adesão a regras consideradas apropriadas, como as difundidas pelo UNICEF para a sociedade global, tais quais os princípios da proteção à criança e ao adolescente.

O Selo consiste basicamente num programa no qual o município deve atender a certos pressupostos e atingir metas de forma a obter a certificação ao final. Embora não haja prejuízo para outras unidades no processo, o programa utiliza a premiação final como incentivo para atingir seus resultados. Assim, ao se apresentar como um incentivo (ganho) final mediante a adoção de determinadas medidas, o Selo acaba se configurando como um mecanismo de competição.

Complementarmente, podemos analisar no trabalho os instrumentos apontados por Jakobi (2009) como utilizados pelas organizações internacionais na difusão de ideias, normas e políticas. É importante ressaltar aqui que, apesar dos instrumentos apresentados pela autora se assemelharem ou mesmo se sobreporem aos mecanismos conforme apresentados por outros autores, a autora considera especificamente o caso das organizações internacionais.

Na visão de Jakobi (2009), o processo se inicia na sociedade global, na qual os fluxos de problemas, políticas e participantes visíveis devem se alinhar para garantir que a ideia central seja bem-sucedida. Na etapa seguinte os instrumentos citados seriam utilizados de forma a influenciar a adoção de determinado tipo de política na esfera interna com o que, em seguida, se obteria mudanças nas políticas internas.

Conforme apresentado anteriormente, o Selo foi uma iniciativa do UNICEF que, em sua primeira edição, abrangia apenas um estado do semiárido brasileiro, o Ceará. O seu sucesso, identificado pelos indicadores avaliados pelo próprio UNICEF, ao fim da primeira edição do programa, ampliou o programa para os 11 estados que fazem parte do semiárido e, posteriormente, para os estados da região amazônica.

Revista Brasileira de Políticas Públicas e Internacionais, v.3, n.2, Dezembro/2018, pp. 152-177. 
OLIVEIRA, Bruna B.; STEINER, Andrea Q.; ALVES, Elia E. C. As organizações internacionais na difusão de políticas de proteção à infância e adolescência no Brasil: um estudo de caso do Selo UNICEF - Município Aprovado

No entanto, antes de aprofundar a questão dos tipos de instrumentos de difusão que o Selo utiliza no âmbito dos municípios, faz-se necessário observar os mecanismos de difusão internacional que o UNICEF utilizou em nível global. Essa etapa inicial possibilitou a existência de um programa como o Selo UNICEF - Município Aprovado, assim como a aceitação que o mesmo tem recebido, havendo adesão de quase a totalidade dos municípios visados pela ação desde a primeira edição.

A questão da infância demorou muito a entrar na agenda internacional, mesmo porque o próprio conceito de infância nos moldes atuais custou a ser reconhecido como tal. Autores como Postman (1999), por exemplo, afirmam que até meados dos séculos XVI e XVII (a depender da região do planeta) as crianças eram vistas como mini adultos e, desde muito cedo, desempenhavam atividades hoje consideradas inapropriadas para essa fase.

Com o início das ações do UNICEF no cenário do pós-Segunda Guerra, dando suporte às crianças em necessidade, a realidade das condições nas quais as mesmas estavam se desenvolvendo em todo o mundo passaram a ser melhor percebidas. Assim, a organização passou a fazer parte permanentemente do corpo da ONU, em 1946, e a organizar fóruns e reuniões com líderes de todo o mundo, nos quais promoviam a ideia de auxílio e melhoria das condições das crianças e jovens de todo o mundo. O World Summit for Children (1990), é um exemplo disso, sendo uma convenção à qual compareceram representantes da maioria dos países. A ocasião serviu para afirmar a situação de risco das crianças em grande parte do mundo, pondo-a como um problema a ser resolvido; este acontecimento pode ser definido com o alinhamento do primeiro fluxo, ou seja, quando dada situação passa a ser percebida como problema, conforme sugerido por Kingdon (1995) e Jakobi (2009).

No que concerne ao alinhamento com o segundo fluxo, o de políticas (formulação de soluções para os problemas), logo em seguida, por ocasião do World Summit for Children de 1990, foi elaborado e assinado pelos 152 países participantes o documento First Call for Children, que punha o desenvolvimento e cuidados com as crianças como prioridade na esfera global. Assim, atingia-se um consenso em relação às intenções dos países no que concerne essa

Revista Brasileira de Políticas Públicas e Internacionais, v.3, n.2, Dezembro/2018, pp. 152-177. 
OLIVEIRA, Bruna B.; STEINER, Andrea Q.; ALVES, Elia E. C. As organizações internacionais na difusão de políticas de proteção à infância e adolescência no Brasil: um estudo de caso do Selo UNICEF - Município Aprovado

parcela da população.

Quanto ao terceiro fluxo (alinhamento dos participantes visíveis e interessados em tomar parte na solução do problema), as vontades conjuntas de diversos atores se alinharam no que diz respeito à elaboração dos Objetivos de Desenvolvimento do Milênio, compromisso assumido em uma cúpula das Nações Unidas em Nova Iorque, em setembro de 2000. Dos objetivos postos na ocasião, seis estavam ligados à criança: erradicar a extrema pobreza e a fome, atingir o ensino básico universal, promover a igualdade entre os sexos e a autonomia da mulher, reduzir a mortalidade na infância, melhorar a saúde materna, combater o HIV/AIDS, a malária e outras doenças, sendo os outros dois garantir a sustentabilidade ambiental e estabelecer uma parceria mundial para o desenvolvimento (UNICEF Brasil, 2017).

Com isso, a ideia de priorizar a infância já se encontrava bem estabelecida. Assim, o Pacto um Mundo para a Criança e o Adolescente do Semiárido já nascia no contexto da Convenção pelos Direitos da Criança, do First Call for Children, e dos Objetivos de Desenvolvimento do Milênio, todos assinados e apoiados pelo Brasil. Dado este fato, passamos a observar quais os mecanismos utilizados pelo UNICEF para incentivar a difusão de políticas públicas para a infância no Brasil.

Todo esse processo já pode ser considerado parte da disseminação de ideias, conforme o primeiro instrumento sugerido por Jakobi (2009). Porém, as ações do próprio programa referente ao Selo também geraram a disseminação de ideias, visto que fez e faz uso de fóruns, seminários e capacitações, além de fazer um acompanhamento dos indicadores sociais dos municípios participantes de forma a incentivar uma melhora na situação da infância e adolescência em seus territórios. Também existem regras e pressupostos que devem ser cumpridos para que os municípios possam participar do programa, tal qual a existência, ou eminente criação, de um conselho municipal da criança e do adolescente.

Outro ponto a se discutir é a questão dos padrões. Relembrando Jakobi (2009), este instrumento promoveria a padronização de comportamentos através de soft e hard law. No que diz respeito aos padrões, tanto o já citado acima, como as diretrizes do Selo UNICEF

Revista Brasileira de Políticas Públicas e Internacionais, v.3, n.2, Dezembro/2018, pp. 152-177. 
OLIVEIRA, Bruna B.; STEINER, Andrea Q.; ALVES, Elia E. C. As organizações internacionais na difusão de políticas de proteção à infância e adolescência no Brasil: um estudo de caso do Selo UNICEF - Município Aprovado

Município Aprovado, encontrados em seu guia metodológico e nos relatórios de resultado das edições, são exemplos de compromissos assumidos pelas partes que uniformizam o comportamento em direção ao objetivo dado pela referida organização internacional.

Em relação aos incentivos financeiros, no caso do Selo pode-se dizer que não há uso deste mecanismo, pois os municípios não recebem nenhum tipo de apoio financeiro direto da organização.

No que diz respeito às funções coordenadoras, o UNICEF realiza monitoramento e divulgação contínua dos indicadores sociais dos municípios que aderem ao Selo, de forma a manter os avanços e regressos sob observação. Esse fato auxilia os municípios a perceber quais as práticas mais bem-sucedidas e quais áreas necessitam de maior atenção. O Selo ainda utiliza fóruns para avaliar a qualidade das políticas que estavam sendo aplicadas nos municípios, de acordo com o relatório do Selo 2006.

O suporte técnico é oferecido na forma de oficinas e consultoria na área de gestão de políticas públicas, área na qual os agentes do UNICEF colaboram para melhorar os programas já existentes nos municípios e na elaboração de novos. Os próprios fóruns acima citados se configuram como instrumento de suporte, pois através deles a organização consegue fornecer conhecimento na área de gestão, além do compartilhamento das experiências que estão sendo levadas a cabo nos diferentes municípios participantes.

Assim, ao considerar esta perspectiva da difusão de Jakobi (2009), pudemos observar a presença de quase todos os instrumentos de difusão utilizados pelas organizações internacionais, à exceção do suporte financeiro. Estes instrumentos seriam aplicados na etapa D do processo de difusão desenhado por ela em seu trabalho, a aplicação dos instrumentos utilizados para promover a etapa E: mudanças nas políticas em nível nacional. Uma síntese destes achados encontra-se no Quadro 02.

Quadro 02. Instrumentos utilizados pelas organizações internacionais na difusão de ideias, normas e políticas (conforme sugerido por Jakobi, 2009) e sua correspondência com o caso do

Revista Brasileira de Políticas Públicas e Internacionais, v.3, n.2, Dezembro/2018, pp. 152-177. 
OLIVEIRA, Bruna B.; STEINER, Andrea Q.; ALVES, Elia E. C. As organizações internacionais na difusão de políticas de proteção à infância e adolescência no Brasil: um estudo de caso do Selo UNICEF - Município Aprovado

Selo UNICEF - Município Aprovado.

\begin{tabular}{|c|c|}
\hline Instrumento & $\begin{array}{l}\text { Observação para o Selo UNICEF - } \\
\text { Município Aprovado }\end{array}$ \\
\hline Disseminação de ideias & $\begin{array}{l}\text { Fóruns, seminários e capacitações; } \\
\text { acompanhamento dos indicadores sociais } \\
\text { dos municípios participantes e incentivo à } \\
\text { melhora; sugestão de regras e pressupostos } \\
\text { para a melhoria das condições das crianças } \\
\text { e jovens; promoção da necessidade de um } \\
\text { conselho municipal da criança e do } \\
\text { adolescente }\end{array}$ \\
\hline Configurações padronizadas & $\begin{array}{l}\text { Diretrizes do Selo encontrados em seu guia } \\
\text { metodológico, que promovem uma } \\
\text { uniformização da forma de agir nas } \\
\text { questões da infância e adolescência; } \\
\text { promoção da necessidade de um conselho } \\
\text { municipal da criança e do adolescente }\end{array}$ \\
\hline Suporte financeiro & ----- \\
\hline Funções coordenadas & $\begin{array}{l}\text { Monitoramento e divulgação contínua dos } \\
\text { indicadores sociais dos municípios que } \\
\text { aderem ao Selo; realização de fóruns para } \\
\text { avaliar a qualidade das políticas aplicadas } \\
\text { para a infância e adolescência }\end{array}$ \\
\hline Suporte técnico & $\begin{array}{l}\text { Oficinas, fóruns e consultoria na área de } \\
\text { gestão de políticas públicas, para melhorar } \\
\text { os programas já existentes nos municípios } \\
\text { e para a elaboração de novos; promoção do } \\
\text { compartilhamento das experiências de } \\
\text { sucesso nos municípios participantes }\end{array}$ \\
\hline
\end{tabular}

Fonte: elaboração própria.

Diante do exposto, podemos observar que o UNICEF faz uso de quatro dos cinco instrumentos de difusão colocados sugeridos por Jakobi (2009). Quanto à difusão de políticas para a infância e adolescência, os relatórios das edições 2005-2006, 2007-2008, 2009-2012 e 2013-2016 do Selo demonstram que houve um aumento nos indicadores de gestão de políticas públicas. Assim, podemos afirmar que a organização internacional estudada agiu, de fato, como

Revista Brasileira de Políticas Públicas e Internacionais, v.3, n.2, Dezembro/2018, pp. 152-177. 
OLIVEIRA, Bruna B.; STEINER, Andrea Q.; ALVES, Elia E. C. As organizações internacionais na difusão de políticas de proteção à infância e adolescência no Brasil: um estudo de caso do Selo UNICEF - Município Aprovado

agente de difusão.

\section{Considerações finais}

O trabalho trouxe a seguinte pergunta de pesquisa: quais os mecanismos de difusão em atuação no processo de ampliação das políticas públicas brasileiras para a infância e adolescência promovidos pelo Fundo das Nações Unidas para a Infância - UNICEF? Foi possível responder plenamente a esta pergunta na medida em que se verificou quatro dos cinco instrumentos de difusão proposto pelo modelo de análise aplicado na pesquisa (Jakobi, 2009), o que mostrou, em última instância, que de fato o UNICEF tem sido um agente de difusão de políticas públicas para a infância e adolescência no Brasil.

Assim, pudemos observar que o UNICEF atuou como difusor de políticas para a infância fazendo uso do programa Selo UNICEF - Município Aprovado para atingir seu objetivo em relação à ampliação de políticas direcionadas à criança no Brasil.

Ainda que haja evidências qualitativas acerca do processo de difusão, em pesquisas futuras será possível aprofundar a análise sobre quais as políticas específicas que foram desenvolvidas em cada município, ou em uma amostra de municípios participantes do projeto. Também poderá ser analisado o papel dos fóruns promovidos pela organização na difusão de políticas entre os municípios, a fim de compreender melhor o funcionamento subjacente ao mecanismo de aprendizagem na difusão de políticas locais através da atuação de organizações internacionais.

Revista Brasileira de Políticas Públicas e Internacionais, v.3, n.2, Dezembro/2018, pp. 152-177. 
OLIVEIRA, Bruna B.; STEINER, Andrea Q.; ALVES, Elia E. C. As organizações internacionais na difusão de políticas de proteção à infância e adolescência no Brasil: um estudo de caso do Selo UNICEF - Município Aprovado

\section{Referências}

Archer, C. (2001) International Organizations. New York: Taylor \& Francis.

Berry, F. S.; Berry, W. D. (1990). State Lottery Adoptions as Policy Innovations: An Event History Analysis. American Political Science Review, vol. 84, n. 2, p. 395-415.

Braun, D.; Gilardi, F. (2006) Taking “Galton's Problem” Seriously -Towards a Theory of Policy Diffusion. Journal of Theoretical Politics, vol. 18, n. 3, p. 298-322.

Gerring, J. (2012) Mere Description. British Journal of Political Science, vol. 42, n. 4, pp. 721746.

Gilardi, F. (2012). Transnational diffusion: Norms, ideas, and policies. In: CARLSNAES, W.; RISSE, T.; SIMMONS, B. (eds.). (2012) Handbook of International Relations. Thousand Oaks: SAGE Publications, p. 453-477.

Gilardi, F. (2016) Four Ways We Can Improve Policy Diffusion Research. State Politics \& Policy Quarterly, vol. 16, n. 1, p. 8-21.

Gourevitch, P. (1978) The Second Image Reversed: The International Sources of Domestic Politics. International Organizations, vol. 32, n. 4, p. 881-912.

Graham, E. R.; Shipan, C. R.; Volden, C. (2012) The Diffusion of Policy Diffusion in Political Science. British Journal of Political Science, vol. 43, n. 3, p. 673- 701.

Heinze, T. (2011). Mechanism-Based Thinking on Policy Diffusion - A Review of Current Approaches in Political Science. KFG Working Paper, vol. 34.

Jakobi, A. P. (2005) The Knowledge Society and Global Dynamics in Education Politics. European Educational Research Journal, vol. 6, n. 1.

Jakobi, A. P. (2009) International organizations and world society: studying global policy development in public policy. TranState Working Papers, vol. 81.

Jakobi, A. P. (2012) International organizations and policy diffusion: the global norm of lifelong learning. Journal of International Relations and Development, vol. 15, n. 1, p. 31-64.

Keohane, R. O.; Nye, J. S. (1987) Power and Interdependence Revisited. International Organization, vol. 41, n. 4, p. 725-753.

Kingdon, J. W. (1995) Agendas, Alternatives, and Public Policies. 2a ed. New York: Logman.

Revista Brasileira de Políticas Públicas e Internacionais, v.3, n.2, Dezembro/2018, pp. 152-177. 
OLIVEIRA, Bruna B.; STEINER, Andrea Q.; ALVES, Elia E. C. As organizações internacionais na difusão de políticas de proteção à infância e adolescência no Brasil: um estudo de caso do Selo UNICEF - Município Aprovado

Knill, C. (2005) Introduction: Cross-national policy convergence: concepts, approaches and explanatory factors. Journal of European Public Policy, Special Issue, p. 764- 774.

Maggetti, M.; Gilardi, F. (2016) Problems (and solutions) in the measurement of policy diffusion mechanisms. Journal of Public Policy, vol. 36, n. 1, p. 87-107.

Maske, T.; Volden, C. (2011) The Role of Policy Attributes in the Diffusion of Innovations. Journal of Politics, vol. 73, n. 1. p. 108-124.

Milner, H. V. (1997) Interests, Institutions and Information: Domestic Politics and International Relations. Princeton: Princeton University Press.

Postman, N. (1999) O desaparecimento da infância. Rio de Janeiro: Graphia.

Putnam, R. D. (1988) Diplomacy and Domestic Politics: The Logic of Two-Level Games. International Organization, vol. 42, n. 3, p. 427- 460.

Simmons, B. A.; Elkins, Z. (2004). The Globalization of Liberalization: Policy Diffusion in the International Political Economy. American Political Science Review, vol. 98, n. 1, p. 171-189.

Simmons, B. A.; Dobbin, F.; Garrett, G. (2006) Introduction: The International Diffusion of Liberalism. International Organization, vol. 60, n. 4, p. 781-810.

UNICEF - UNITED NATIONS CHILDREN’S FUND. (1990) First Call for Children.

UNICEF - UNITED NATIONS CHILDREN'S FUND. (2018) Site institucional. Disponível em <http://www.unicef.org.br>. Acesso em 30 setembro 2018.

UNICEF Brasil. (2017a). Objetivos de Desenvolvimento do Milênio. <http://www.unicef.org/brazil/pt/overview_9540.htm>. Acesso em 28 abril 2017. 2017a.

UNICEF Brasil. (2017b). Selo UNICEF Município Aprovado. Disponível em <http://selounicef.org.br/>. Acesso em 28 abril 2017.

Volden, C. (2006) States as Policy Laboratories: Emulating Success in the Children's Health Insurance Program. American Journal of Political Science, vol. 50, n. 2, p. 294-312.

Weyland, K. G. (2005) Theories of Policy Diffusion: Lessons from Latin American Pension Reform. World Politics, vol. 57, n. 2, pp. 262-259.

Yin, R. K. (2009). Case study research: design and methods. Sage Publications, 4a ed. California, USA. 240p.

Revista Brasileira de Políticas Públicas e Internacionais, v.3, n.2, Dezembro/2018, pp. 152-177. 
OLIVEIRA, Bruna B.; STEINER, Andrea Q.; ALVES, Elia E. C. As organizações internacionais na difusão de políticas de proteção à infância e adolescência no Brasil: um estudo de caso do Selo UNICEF - Município Aprovado

\section{Documentos da ONU e UNICEF utilizados na análise}

UN - UNITED NATIONS. 1946 General Assembly. Establishment of an International Children's Emergency Fund.

UNICEF - UNITED NATIONS CHILDREN'S FUND Documento de Programa do País revisado - Brasil (2012-2016).

UNICEF - UNITED NATIONS CHILDREN'S FUND Final Report of the First Executive Board of the United Nations International Children's Emergency Fund - 11 December 1946-31 December 1950.

UNICEF - UNITED NATIONS CHILDREN’S FUND. Guia Metodológico - Selo Município Aprovado - Edição 2013-2016/ Semiárido.

UNICEF - UNITED NATIONS CHILDREN'S FUND. Resultados do Selo UNICEF Município Aprovado - Edição 2008.

UNICEF - UNITED NATIONS CHILDREN'S FUND. Selo UNICEF Município Aprovado Amazônia - Resultados do Selo 2009/ 2012.

UNICEF - UNITED NATIONS CHILDREN'S FUND. Selo UNICEF Município Aprovado Resultados de dois anos de implementação no Semiárido brasileiro (2005/2006).

UNICEF - UNITED NATIONS CHILDREN'S FUND. Selo UNICEF Município Aprovado Semiárido - Resultados do Selo 2009/ 2012.

UNICEF - UNITED NATIONS CHILDREN'S FUND. Strategies for Children for the 1990's.

UNICEF - UNITED NATIONS CHILDREN'S FUND. The contribution of UNICEF to the peace process

UNICEF - UNITED NATIONS CHILDREN'S FUND. UNICEF Annual Report 2013 - Brazil.

Revista Brasileira de Políticas Públicas e Internacionais, v.3, n.2, Dezembro/2018, pp. 152-177. 